\title{
Normal Pressure Hydrocephalus: A Simple Hypothesis*
}

\author{
Gardar Gudmundsson \\ Uppsalir Medical Center, Reykjavik, Iceland \\ Email: gardar@grusk.is
}

Received October 21, 2012; Revised December 4, 2012; accepted December 24, 2012

\begin{abstract}
Normal pressure hydrocephalus is a devious phenomenon. It is a disease that is difficult to diagnose and difficult to treat, the only treatment being a ventriculo-peritoneal shunt, though good shunting results rarely pass a $70 \%$ level of effectiveness. We need to understand its pathophysiology better before things will improve. Although some colleagues know it as a possible "reversible dementia" others hardly know about its existence. Solutions would also have value for the general understanding of hydrocephalus of other types. Many theories have been published recently in the search for the missing pieces in this puzzle and I feel that my own postulations could turn out to be useful. After years of diagnosing and operating on hydrocephalus patients I propose that: 1) There is reason to believe that patients with the Apoprotein E3/3 genotype and a high head size percentile are particularly vulnerable to developing idiopathic normal pressure hydrocephalus (iNPH). 2) The classical theory that the arachnoid granulations (AG) transport cerebrospinal fluid (CSF) into the venous circulation is wrong. I postulate, that the AG essentially are sensors, registering the pressure differences between the CSF in the subarachnoidal space at the top of the skull and the venous pressure in the sagittal sinus. The AG's endothelium produces nitric oxide (NO) as a messenger that is received by the vagus nerve at the jugular foramen. 3) The disease has its fundamental pathology in the subpial space in the large cisternas and along the large vessels under the brain. Here the intravenous absorption of cerebrospinal fluid (CSF) takes place. Cerebrospinal fluid is transported into the subpial venules and veins, driven by the pulse pressure of the subpial arteries. Morphological changes in the pial/subpial anatomy explain the existence of acquired normal pressure hydrocephalus (aNPH).
\end{abstract}

Keywords: Normal Pressure Hydrocephalus; ApoE3; Head Size; Arachnoid Granulations; Subpial Absorption

\section{Introduction}

Our brains float in water, but it has to be the right amount and under the right pressure. In spontaneous intracranial hypotension, an opening in spinal parts of the dura lets CSF slip out and the patients get a headache that is worst in the upright position but can disappear when lying down. If this situation is not reversed, bilateral paresis of the abducens nerve can occur, subdural hematomas can develop, and even disturbance of consciousness.

The diagnosis hydrocephalus on the other hand is "too much water in the brain", and has been used for over 300 years to describe a disease where the damaging effects of disturbed resorption of CSF causes affected infants to grow large heads and suffer damage to the brain itself and often an early death before the time of shunt insertions. Gradually it became known that hydrocephalus could also develop later in life, after hemorrhages, infections, trauma and operations, and also with closure of the cerebral aqueduct. From this knowledge two types of hydrocephalus were defined: communicating and obstructive.

\footnotetext{
${ }^{*}$ Conflicts of interest: None declared.
}

In 1965 Dr. Salomon Hakim of Bogotá wrote an article in Spanish about a new neurological disease with enlargement of the ventricles of the brain and three cardinal clinical symptoms: impaired gait, incontinence and dementia. He measured the intracranial pressure (ICP) to be within normal limits, but by withdrawing some CSF the patients improved. This led him to develop a shunt, a device for allowing spinal fluid to flow from the ventricles down into the abdominal cavity. He called this disease "normal pressure hydrocephalus", a communicating hydrocephalus. The same year an article in English by Hakim as a coauthor presented his work to the Englishreading population [1].

In the decades since Hakim's discovery we have made some progress. Still there are issues that are disturbing.

In 2005 guidelines for the diagnosis and management of idiopathic normal pressure hydrocephalus were published in the journal Neurosurgery. Leading experts had met and evaluated the known facts. Their conclusion was that iNPH diagnostic work can only lead to a probable, possible or unlikely diagnosis. They agreed that a Tap Test (approx. $40 \mathrm{ml}$ of spinal fluid removed by lumbar puncture) was useful only when it was positive, when it 
could lead to improvements in clinical symptoms. However, it was of little value when negative, but a prolonged CSF drainage (over $300 \mathrm{ml}$ ) had good specificity and sensitivity [2].

Harold O. Conn a former Yale Medical School faculty member, developed iNPH from 1992 until diagnosed in 2002. During this time he was diagnosed and treated for other better known diseases without any improvement. After having a shunt implanted he made good recovery. Recognizing the lack of awareness for NPH among physicians, he interviewed 166 practicing colleagues and found out that almost one-third had never heard about NPH. In 2005 he repeated his survey with 188 Yale Medical School graduates and found out that two-thirds had learned about NPH in medical school, and $7 \%$ had never heard of it [3].

We are confronted by an important disease where treatment can reverse dementia but we are not able to diagnose it accurately, treatment often fails and many colleagues are not aware of its existence. Due to the diagnostic difficulties, research is probably disturbed by "mixed" groups of patients.

\section{The ApoE3 Theory}

In a small study published in June 2009 [4] we examined 15 patients who were referred consecutively to a neurosurgical unit from neurologists and geriatricians as likely to have iNPH. The patients all had at least two of the three cardinal symptoms that form the NPH syndrome: impaired gait, urinary incontinence and dementia, not to mention the fact that all were elderly. CT scans or MRI of the head had shown enlarged ventricles. We chose a Tap Test in an effort to find those most likely to benefit from a shunt operation.

The relative change in walking speed after the Tap Test is shown in a box plot in Figure 1.

All those who had a positive Tap Test were of the ApoE3/3 genotype and had large heads.

The ApoE3 homozygotes all increased their walking speed considerably, all had over a $20 \%$ increase, with a mean value of $35 \%$ speed increase, whereas the other ApoE genotypes formed a distribution with a mean value of $0 \%$, all with less than $20 \%$ values. These results were statistically significant.

\section{Head Size}

Although brain size is largely determined by genetics, lifestyle and developmental issues also play a role. The connection between iNPH and large head size has been postulated by Wilson and Williams at Johns Hopkins Hospital, Baltimore, among others. In their article they conclude with the statement, that "a significantly larger proportion of elderly adults with iNPH have a head cir-

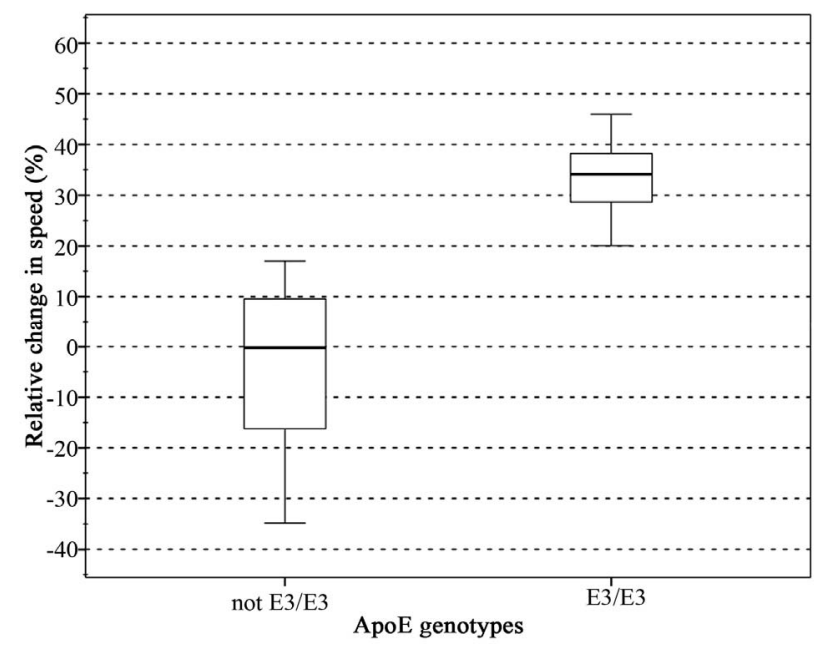

Figure 1. ApoE3 homozygotes (the group on the right) form a distinct group with a mean value around $35 \%$ relative speed change in the Tap Test. The rest (the non ApoE3/3, the group on the left) formed a group with a mean value of zero.

cumference greater than the 90th or 97th percentile than predicted by population norms" [5]. They see iNPH partially as a compensated congenital hydrocephalus that becomes symptomatic late in life.

Bradley and coworkers have come to a similar conclusion, that patients with NPH have an intracranial volume significantly larger than normal. An initial insult must have taken place before the close of sutures, according to their theory [6]. The idea of a naturally occurring large head is not taken into account.

The frequency of the ApoE3/3 genotypes varies widely in the literature, with values from $45 \%$ - $75 \%$ frequent. If we assume the true value to be about $60 \%$ and all the iNPH patients to be over the 90th percentile in head size, we end up with $6 \%$ of the population. The actual number of iNPH patients is unknown, as can be expected when the diagnostic process is still not defined, but if the hypothesis holds true, then possibly $6 \%$ of the population are at risk of developing iNPH when arteriosclerosis sets in.

\section{Arachnoid Granulations}

In some animals the arachnoid granulations (AG) do absorb spinal fluid, but in humans this has never been clearly demonstrated.

But if we have been mistaken all these years and CSF absorption takes place in the subpial spaces, mostly in the large cisternas under the brain, what function do we give to the AG? My feeling is that an analogy can be drawn from another ectodermal tissue: the epidermis. Here we have an ectodermal germ layer that lies against another germ layer with many kinds of sensors implanted. AG could be sensors that react to changes in pressure 
between the ICP and the venous pressure, thereby giving signals regarding the pressure at the top of the skull. We are generally lacking in understanding how venous pressure is controlled inside the skull and the AG first develop as we learn to sit, stand and walk, a big difference from our position in the first part of life, mostly spent lying flat. My thought is that the AG react to changes in venous pressure versus ICP, mostly due to positional changes, by producing a chemical substance that is registered at another location. The simplest form would be nitrous oxide (NO) production by the endothelial covering of the AG which would be registered at the jugular bulb, just as the venous blood flow leaves the skull. Here the vagal nerve is in a position to receive the message and translate the information to the central circulation.

The conclusion can also be drawn from this theory that with increasing ICP and normal control on the intracranial venous pressure, the ventricles will dilate. If the venous control is disturbed, the ventricles are less likely to dilate, as is sometimes seen in meningitis and is the rule in pseudotumor cerebri and might be because the AG are not functioning properly.

The simplest form for control in venous intracerebral blood flow would be by viscosity changes in the smaller vessels, as their diameter cannot be controlled.

\section{The Subpial Theory}

R.O. Weller and colleagues in Southampton, UK, published in 2009: "Lymphatic drainage of the brain and the pathophysiology of neurological disease”. In Weller's article, the authors draw a line between CSF and interstitial fluid (ISF) of the brain. Where the ISF comes from the blood and the metabolic activity of the brain tissue, the ISF then drains out with solutes into the circulation. This takes place in the perivascular and subpial spaces of the brain [7]. Bulat and coworkers in Croatia showed in 2008 that CSF and ISF are in continuity, that they are 99\% water and are reabsorbed into venous capillaries and postcapillary venules [8].

This fits with the idea that ISF and CSF behave like lymph fluid in other organs, where the absorption is never into arteries but only into venous structures.

Sakka et al. wrote in 2011 a review article about the anatomy and physiology of cerebrospinal fluid [9]. Although they regard the mechanism regulating cerebrospinal fluid pressure as not fully elucidated, it is determined by parenchymal and venous pressures. They believe that CSF in humans is partially absorbed along the optic, trigeminal, facial and vestibulocochlear nerves, the olfactory mucosa, spinal nerve sheaths and in the brain via Virchow-Robin perivascular spaces. Thus CSF enters the lymphatic system.

In sheep the occlusion of the cribiform plate raises ICP and increases CSF absorption into the lymphatic system by other routes.

Recently it was demonstrated that in mice brains interstitial fluid is cleared along paravenous drainage pathways [10].

This also points to the fact that hydrocephalus is often a relative situation, that there is not enough CSF absorbed, but rarely a complete stop. As neurosurgeons know, a patient with a massive subrachnoidal hemorrhage may acutely need an external drainage in order to survive, as his basal cisternas are filled with blood, whereas an NPH patient can wait for days for the operation. In the acutely sick patient all flow out of the ventricles may be blocked, but in the NPH patient there is diminished absorption in the subpial spaces due to less pulse pressure and other routes for CSF absorption may be functioning normally, though with relatively diminished absorption.

According to the subpial theory, the arteries in the subpial space are without the coverings they have in the subarachnoidal space and their distensions with every heartbeat in the tightly packed subpial space drives CSF into venules and veins. The CSF enters the subpial spaces from the subarachnoidal space either through diffusion or through small pores.

It seems that ISF has more routes to enter the venous system, in the brain parenchyma and in the subpial spaces over the hemispheres.

In $2001 \mathrm{M}$. Egnor et al. postulated that "arterial pulsations are dissipated through the CSF to the cerebral veins, bypassing the capillary circulation" [11].

In 2006 Japanese scientists seeking to establish the relation between the pulsatile components of pressure and flow waveforms in the carotid artery and their change with age concluded that:

"Although it is accepted that upper-body flow waveforms are completely different from wave patterns in the ascending aorta and in lower-body arteries, such waves are known to show considerable variability in normal human subjects, their relation with the carotid pressure wave and with subsequent disease has not been considered" and also that age related arterial stiffening can explain the increasing flow fluctuations in cerebral blood vessels [12]. Reduced pulse pressure in the carotid arteries would then reduce absorption of CSF.

The periventricular hypodensity seen on CT scans in NPH probably results from CSF entering the brain parenchyma from the basal cisternas and through the pia and glia limitans and distributing in the less dense area of the brain, a sign of disturbed absorption of CSF into the subpial venous circulation. 


\section{Conclusions}

This article presents a hypothesis, a model for observable but unexplained facts, but also "progressive focusing", a qualitative method where some experimental facts are used in an effort to understand a disease that is not fully charted. The hypothesis has evolved during the search for a meaningful explanation to known problems regarding the normal pressure hydrocephalus diagnosis [13].

The conclusion is that the pathology regarding CSF absorption lies in the subpial spaces in the basal cisternas and along the great vessels entering the brain. Here the idiopathic NPH is caused by reduced pulse pressure, which is needed to press the CSF into the venules. Here the ApoE3/3 genotype with the largest head percentile seems to be most vulnerable. For the acquired type of NPH the disruption of the subpial space reduces the entrance and subsequent resorption of CSF. The arachnoid granulations play no role in resorption of CSF, but are rather most likely sensory and secretory organs necessary to control intracranial venous pressure.

If this new model is proven to be correct, our view of disturbed CSF absorption in NPH and regular hydrocephalus will change and hopefully new therapies will develop.

First adequately designed studies are needed to confirm or reject this theory.

\section{The Hypothesis Includes the Following Statements}

1) Elderly patients with the ApoE3/3 genotype and belonging to a percentile with large heads are most likely to develop iNPH when their pulse pressure lowers.

2) The AG are sensory/secretory and produce NO with changes in the pressure differences between the ICP and the venous pressure inside the sagittal sinus.

3) The NO is registered by the vagal nerve at the jugular foramen.

4) Venous pressure is probably controlled by changing viscosity in smaller veins.

5) Normal venous control is necessary for the ventricles to dilate with increasing ICP.

6) The pulse pressure in the cerebral subpial arteries is the driving force for the venous absorption of CSF.

7) Absorption of CSF into the venous circulation mainly takes place in the subpial space ventrally, in the large cisternas and along the large vessels. This absorptive area is vastly larger than the alleged absorptive area of the arachnoid granulations. Scientists find it likely that a part of the CSF in humans is also absorbed along cranial and spinal nerves and into veins in the Vircow-Robin spaces.

8) Morphological changes in the subpial spaces or the pia itself explain aNPH.
9) The absorption of ISF is probably along venous structures in the brain itself and in the subpial spaces over the cerebral hemispheres.

10) The changes seen on CT in NPH, called periventricular hypodensity, are caused by CSF that has entered the brain from the basal subpial spaces and diffused along the tracts of the brain parenchyma, excluded from the basal nuclei because of their density.

\section{REFERENCES}

[1] R. D. Adam, C. M. Fisher, S. Hakim, et al., "Symptomatic Occult Hydrocephalus with 'Normal' Cerebrospinal Fluid Pressure. A Treatable Syndrome,” The New England Journal of Medicine, Vol. 273, 1965, pp. 117-126. doi:10.1056/NEJM196507152730301

[2] N. Relkin, A. Marmarou, P. Klinge, M. Bergsneider and P. M. Blach, "Diagnosing Idiopathic Normal-Pressure Hydrocephalus,” Neurosurgery, Vol. 57, 3 Suppl., 2005, pp. 4-16, Discussions ii-v.

[3] H. O. Conn and F. M. Lobo, "What Do Physicians Know about Normal Pressure Hydrocephalus and When Did They Know It? A Survey of 284 Physicians,” Yale Journal of Biology and Medicine, Vol. 81, No. 1, 2008, pp. 19-29.

[4] G. Gudmundsson, G. Kristjansdottir, E. Cook and I. Olafsson, "Association of ApoE Genotype with Clinical Fea tures and Outcome in Idiopathic Normal Pressure Hydrocephalus (iNPH): A Preliminary Report,” Acta Neurochirurgica, Vol. 151, No. 11, 2009, pp. 1511-1512. doi:10.1007/s00701-009-0429-8

[5] R. K. Wilson and M. A. Williams, "Evidence That Congenital Hydrocephalus Is a Precursor to Idiopathic Normal Pressure Hydrocephalus in Only a Subset of Patients," Journal of Neurology, Neurosurgery \& Psychiatry, Vol. 78, No. 5, 2007, pp. 508-511. doi:10.1136/jnnp.2006.108761

[6] W. G. Bradley, F. G. Safar, C. Hurtado, J. Ord and J. F. Alksne, "Increased Intracranial Volume: A Clue to the Etiology of Idiopathic Normal-Pressure Hydrocephalus?” American Journal of Neuroradiology, Vol. 25, No. 9, 2004, pp. 1479-1484.

[7] R. O. Weller, E. Dujanda, H.-Y. Yow and R. O. Carare, "Lymphatic Drainage of the Brain and the Pathophysiology of Neurological Disease,” Acta Neurochirurgica, Vol. 117, No. 1, 2009, pp. 1-14. doi:10.1007/s00401-008-0457-0

[8] M. Bulat, V. Lupret, D. Oreskovic and M. Klarica, “Transventricular and Transpial Absorption of Cerebrospinal Fluid into Cerebral Microvessels," Collegium Antropologicum, Vol. 32, Suppl. 1, 2008, pp. 43-50.

[9] L. Sakka, G. Coll and J. Chazal, "Review of the Literature. Anatomy and Physiology of Cerebrospinal Fluid,” European Annals of Otorhinolaryngology, Head and Neck Diseases, Vol. 128, No. 6, 2011, pp. 309-316.

[10] J. J. Iliff, M. Wang, Y. Liao, B. A. Plogg, W. Peng, G. A. Gundersen, H. Benveniste, G. E. Vates, R. Deane, S. A. Goldman, E. A. Nagelhus and M. Nedergaard, "A Para- 
vascular Pathway Facilitates CSF Flow through the Brain Parenchyma and the Clearance of Interstitial Solutes, Including Amyloid $\beta$,” Science Translational Medicine, Vol. 4, No. 147, 2012, Article ID: 147ra111.

[11] M. Egnor, A. Rosiello and L. Zheng, "A Model of Intracranial Pulsations,” Pediatric Neurosurgery, Vol. 35, No. 6, 2001, pp. 284-298. doi:10.1159/000050440
[12] K. Hirata, T. Yaginuma, M. F. O’Rourke and M. Kawakami, "Age-Related Changes in Carotid Artery Flow and Pressure Pulses: Possible Implications for Cerebral Microvascular Disease,” Stroke, Vol. 37, No. 10, 2006, pp. 2552-2556. doi:10.1161/01.STR.0000242289.20381.f4

[13] T. Greenhalgh, "How to Read a Paper," 4th Edition, BMJ Books, London, 2010, pp. 166-167. 